\title{
Partial word knowledge in the absence of recall
}

\author{
Alan S. Brown - Christopher N. Burrows • \\ Kathryn Croft Caderao
}

Published online: 4 May 2013

(C) Psychonomic Society, Inc. 2013

\begin{abstract}
Attributes of words can be known even when the words are not currently retrievable. Although repeatedly demonstrated for semantic and contextual dimensions, the evidence is ambiguous for structural characteristics. The present research demonstrates significant above-chance first-letter knowledge across four ordinal levels of retrieval confidence for nonretrieved words - tip of the tongue (TOT), high familiar, low familiar, unfamiliar. Contrary to prior research, there was minimal evidence for syllable number knowledge, even at highest confidence levels. Initial letter recognition in the absence of retrieval resembles the recognition without identification in episodic memory (Cleary, Current Directions in Psychological Science 17: 353-357, 2008), and such implicit familiarity may contribute more generally to confidence assessments of word knowledge in both semantic and episodic memory domains. Furthermore, this outcome suggests that word feature priming in the form of partial phonological activation may occur to some extent for all words during a retrieval attempt, and even for ones that are judged to be unknown.
\end{abstract}

Keywords Implicit memory $\cdot$ Memory $\cdot$ Word production · Metamemory · Tip of the Tongue

Retrieval of known information is frequently fragmental, where certain contextual or semantic attributes are available even when the fact or name is not (Koriat, Levy-Sadot, Edry, \& de Marcas, 2003). The possibility of word feature

A. S. Brown $(\bowtie) \cdot$ C. N. Burrows $\cdot$ K. Croft Caderao Department of Psychology, Dedman College,

Southern Methodist University, Dallas, TX 75275, USA

e-mail: abrown@smu.edu

\section{A. S. Brown}

Department of Psychology, Dedman College,

Southern Methodist University, Dallas, TX 75274, USA availability in the absence of word retrieval was first empirically demonstrated by Brown and McNeill (1966) in their study of the tip of the tongue (TOT) experience (Brown, 1991, 2012; Schwartz, 2002). This is perhaps the most salient subjective experience associated with TOTs-the availability of semantic and structural information about the word (e.g., first letter, number of syllables) - despite the failure to retrieve it. Such attribute information availability has been central to theoretical speculation and debate about TOTs. From the direct access theoretical perspective, this information reflects a subthreshold excitation that is sufficient to allow access to target word features but inadequate for whole-word production (transmission deficit hypothesis, or TDH; Burke, MacKay, Worthley, \& Wade, 1991). Alternatively, the metacognitive (inferential) perspective assumes that such information drives one's subjective evaluation of eminent retrieval, apart from any activation of the target word itself (Koriat, 1993; Metcalfe, Schwartz, \& Joaquim, 1993; Schwartz, 2002). The general assumption of both theoretical perspectives is that access to target word features in the absence of its retrieval is associated only with the highest confidence retrieval states (i.e.,TOT).

The purpose of the present investigation is to determine to what extent such information is also accessible during retrieval attempts where one is not so sure about the retrievability of the sought-after word. There is intriguing evidence that semantic dimensions of unretrievable words (e.g., semantic differential) can be identified in the absence of retrieval certitude (Eysenck, 1979; Koriat et al., 2003; Yavuz \& Bousfield, 1959) and has even been observed when the nonretrieved word is unknown or judged to be a nonword (Durso \& Shore, 1991; Shore \& Durso, 1990). In contrast, such access for structural or orthographic features of nonretrieved words has not been clearly demonstrated for states other than high retrieval certitude (TOTs).

Also related to this issue is the recognition without identification (RWI) phenomenon, where one can be aware of 
attributes of a stimulus in the absence of consciously available knowledge regarding what this information is. RWI was originally demonstrated by Peynircioğlu (1990), who gave participants an input list of words to study, followed by a test using word fragment cues. If unable to complete the fragment, participants had to guess whether the word that completed the fragment had appeared on the prior input list. Participants were above chance on such assessments, suggesting that the fragment implicitly elicited familiarity in the absence of explicit recollection of the earlier encounter. Some feature of the test cue was connected to the episodic memory representation, giving rise to a sense of familiarity for that cue. The finding has been subsequently replicated across a wide variety of cue materials, including odors (Cleary, Konkel, Nomi, \& McCabe, 2010), music (Kostic \& Cleary, 2009), famous landmarks (Cleary \& Reyes, 2009), simple line drawings (Cleary, Langley, \& Seiler, 2004), and celebrity faces (Cleary \& Specker, 2007). For example, participants are able to identify that a famous face shown at test had the associated name presented previously during input (above chance), even though they cannot retrieve the name (Cleary \& Specker 2007).

Although Cleary's RWI research was limited to episodic memory tasks, the same mechanisms may apply to assessing the availability of structural word information from longterm memory. The RWI research deals primarily with the recency attribute (does the current stimulus relate to another recently experienced stimulus?), but the same processes could also operate in detecting other attributes associated with the lexical memory representation (first letter, part of speech). The automatic priming of such information may be a key component underlying confidence ratings and TOT assessments. In fact, Cleary has likened the RWI phenomenon to the TOT experience (Cleary, 2006; Cleary et al., 2010; Cleary \& Reyes, 2009; Cleary \& Specker, 2007), assuming that sensing that a word is stored in semantic memory may be similar to feeling that a word appeared in the prior list.

In fact, several investigations evaluated whether RWI was driven solely by a TOT experience related to a prior list presentation. Studies involving definitions (Cleary, 2006), famous faces (Cleary \& Specker, 2007), and famous scenes (Cleary \& Reyes, 2009) consistently found that RWI and TOT are separable: RWI is found for both TOT and don't know (DK) items. It would seem to be a reasonable extension of this research to assume that features of an inaccessible word other than recency (i.e., orthographic, structural) could also be available in the absence of retrieval, including those associated with low confidence of subsequent identification (DK).

In order to gain a more accurate assessment of access to information during the DK state, the present research provided a more fine-grained differentiation across levels of
DK by using rating categories of unfamiliar (or UF), low familiar (or LF), and highly familiar (or HF). The reason for making this finer differentiation at the high end of DK (HF) is that there may be some permeability in that weaker TOTs may be classified with DKs (Bahrick, 2008; Jönsson \& Olsson. 2003; Jönsson, Tchekhova, Lönner, \& Olsson, 2005; Naito \& Komatsu, 1989; Schwartz, 2002; Schwartz, Travis, Castro, \& Smith, 2000). Indeed, a few investigations have revealed some first-letter (phoneme) knowledge for DK items (Beidermann, Ruh, Nickels, \& Coltheart, 2008; Caramazza \& Miozzo, 1997; Gollan \& Acenas, 2004; Koriat \& Lieblich, 1974; Miozzo \& Caramazza, 1997; Schwartz, 2008; Smith, Balfour, \& Brown, 1994), but one could argue that participants classified some weak TOTs as DKs and that such knowledge does not exist for moderate or low-confidence DKs.

We assume that if there are weak TOTs that do not pass the subjective threshold for TOT, participants should relegate such items to the highest DK classification (HF). Thus, if the modest evidence for first-letter identification success for DK items (Koriat \& Lieblich, 1974) is driven by those weak TOTs mixed in with DKs, first-letter access should be evident only for HF items. On the other hand, if first-letter access exists more generally for all DK items, above-chance performance should be found across UF, LF, and HF items.

To preview the present investigation, picture cues were used to assess participants' access to structural features for unretrieved target words (first letter; syllable number) across four levels of retrieval confidence. Word feature guesses consisted of selecting the correct letter from among three choices (Experiments 1 and 2) and choosing the number of syllables from among five (Experiment 1) or two (Experiment 2) choices. Both recognition decisions were made immediately following retrieval confidence assessment for each unretrieved word, and the order was alternated from Experiment 1 (letter, syllable) to Experiment 2 (syllable, letter).

\section{Experiment 1}

Method

Participants

A total of 37 students in sections of Introductory Psychology at Southern Methodist University volunteered to participate in the study and received extra course credit as incentive.

\section{Materials}

Black-and-white and color pictures were selected from a variety of sources, including dictionaries, published norms 
(Snodgrass \& Vanderwart, 1980), and the Web. Starting with an initial set of over 300 stimuli, several pilot studies helped identify those 127 target words with a picture-cued recall probability of between $25 \%$ and $78 \%$. These pictures were then uniformly sized for PowerPoint presentation. A list of labels for all cue-object pictures appears in Table 1.

A set of three letters was constructed for each target word, consisting of its first letter plus two other letter lures. These letter sets were constructed using the Mayzner and Tresselt (1965) norms, which lists the frequencies with which each single letter and letter pair appears in a sample of 20,000 written words. These frequencies are listed separately for each position within the word, and separately for words of lengths three through seven letters long. From these norms, we used only the probability of occurrence for single letters in the initial position of words. We rank ordered all letters from high to low in terms of the frequency with which they appear as initial letters. The two incorrect (lure) letters for each target word were selected from a five-letter bracket, formed by selecting the two letters that ranked immediately higher and the two that fell immediately lower than the first letter for that target word. This was done so that frequency discrimination among the letters would be relatively difficult and any bias to consistently guess the relatively higher or lower ranked initial letter would not be successful.

To illustrate, "C" falls in the middle of the rank-ordered letters (high to low) B, M, C, O, and L. Thus, the 16 target words beginning with the letter $\mathrm{C}$ used three-letter combinations such as CBM, OCB, and LOC. Letter order within cue sets was randomized, with the restriction that the correct first letter appeared approximately equally often in each of the three positions across items (see Table 1). The cue-letter set for each target remained constant across participants. Picture cues were randomly assigned to presentation position, which also remained constant across all participants.

\section{Procedure}

Participants were tested individually with PowerPoint software on a PC computer, and an experimenter recorded their oral responses. They were told that pictured objects would appear on the screen, one at a time, and that they should name the object if they could. Participants were allowed up to $15 \mathrm{~s}$ to retrieve the target label. If they could not, they were asked to indicate how familiar they were with the object's name:

unfamiliar (UF): I have no knowledge of what the word is.

low familiar (LF): I have come across this word before and might recognize it.

high familiar (HF): I would probably recognize the word.
Table 1 Target words and letter cue sets used in Experiments 1 (all)

and $2(*)$

abacus*

T A S

accordion

T A W

acupuncture

A T W

anteater*

T S A

anvil*

A T S

artichoke*

W T A

awning*

W S A

backgammon* B M C

bagpipe*

F B H

barometer

C M B

bazooka*

M F B

blimp

bonsai*

Braille

bulldozer*

caboose

candelabra

carabineer

castanets*

chaps

chariot*

clavicle*

cobra*

colander*

comet*

croquet*

crossbow*

crowbar*

crow's nest

curling*

cymbals*

doily*

dominoes*

drawbridge*

dumbbell*

easel*

éclair*

eclipse

eel

fire hydrant*

fossil*

French horn*

gallows

gargoyle*

gavel*

Geisha*

gondola*

guillotine*

harmonica

harp
B M H

$\mathrm{H}$ B M

B C F

$\mathrm{H}$ B C

B M C

O M C

M C L

O L C

$\mathrm{B} \mathrm{CO}$

$\mathrm{CO} B$

$\mathrm{M} \mathrm{CO}$

$\mathrm{COM}$

L B C

C L B

$\mathrm{C}$ M B

$\mathrm{M} \mathrm{O} \mathrm{C}$

$\mathrm{L} \mathrm{O} \mathrm{C}$

$\mathrm{C} M \mathrm{~L}$

$\mathrm{M} \mathrm{C} B$

L C B

P R D

D N R

N P D

L D N

E I K

Y K E

G E I

G Y E

$\mathrm{MH} \mathrm{F}$

S F M

F B S

D Y G

N E G

R G N

R G E

G Y R

G Y E

H F B

W F H 
Table 1 (continued)
Table 1 (continued)

\begin{tabular}{|c|c|}
\hline harpoon* & W S H \\
\hline hexagon* & S H B \\
\hline hinge & C H D \\
\hline hoe & $\mathrm{HW} \mathrm{S}$ \\
\hline honeydew* & B H W \\
\hline hourglass* & B S H \\
\hline hypotenuse & H S F \\
\hline icicle* & Y U I \\
\hline igloo* & E I U \\
\hline kilt & K E V \\
\hline kiwi* & $\mathrm{C} \mathrm{K} \mathrm{O}$ \\
\hline lacrosse* & L D P \\
\hline level & L O D \\
\hline life preserver & P C L \\
\hline llama & O L P \\
\hline mammoth* & $\mathrm{COM}$ \\
\hline maracas* & F B M \\
\hline metronome* & M C F \\
\hline minaret* & $\mathrm{M} \mathrm{C} \mathrm{O}$ \\
\hline monkey wrench* & M O B \\
\hline moose & B M C \\
\hline Morse code* & $\mathrm{F} \mathrm{M} \mathrm{O}$ \\
\hline muzzle* & O F M \\
\hline observatory & O P L \\
\hline origami & F O M \\
\hline ouija board* & M P O \\
\hline palate* & P H F \\
\hline parallel bars & L P D \\
\hline Parthenon* & O L P \\
\hline pomegranate & O R P \\
\hline porcupine* & P O R \\
\hline pulley* & O P R \\
\hline pitchfork* & D P O \\
\hline Pegasus* & L R P \\
\hline periscope* & R P D \\
\hline platypus* & P D O \\
\hline quiver & S Q W \\
\hline radiator & R D G \\
\hline rickshaw* & P R G \\
\hline safety pin* & F S H \\
\hline Saturn & S H F \\
\hline saxophone* & $\mathrm{HAS}$ \\
\hline scorpion* & S A W \\
\hline seahorse* & W S H \\
\hline seismograph* & W S A \\
\hline sextant* & H W S \\
\hline siphon & S H W \\
\hline sloth & H F S \\
\hline spatula* & $\mathrm{COS}$ \\
\hline sphinx & S H A \\
\hline
\end{tabular}

\begin{tabular}{ll} 
spinning wheel* & W S F \\
spur & F W S \\
stethoscope* & S F W \\
stilts & A S F \\
stingray* & F A S \\
stock(ade) & S F A \\
Stonehenge* & W S H \\
sundial* & A W S \\
swastika* & S H A \\
tadpole* & T W S \\
tambourine & A T H \\
telescope* & T G R \\
thimble* & N T A \\
tongs & F T M \\
totem pole* & T W B \\
toucan* & W A T \\
trampoline* & T C L \\
treble clef* & D P T \\
tripod* & B T N \\
trombone* & D F T \\
turnstile & H GT \\
unicorn* & K U V \\
vice & V R L \\
walrus* & T W H \\
weathervane* & W T S \\
xylophone* & Y V X \\
yoke & H A Y \\
\hline & \\
\hline
\end{tabular}

tip of the tongue (TOT): I am on the verge of recalling it; it is in my mind, but I can't quite get it out at the moment.

These instructions appeared on a PowerPoint slide and were reviewed with the experimenter to be sure that the participants understood the response categories. In addition, the categories and definitions were placed in front of the participants on a sheet of paper so that they could refer to them throughout the procedure.

Participants were further instructed that after their familiarity rating, three letters would appear in the upper left corner of the picture, one of which was the first letter of the pictured object's name. If they had produced the target word, they were instructed to simply ignore this and were told that the next object cue would be presented. If they did not provide the target word (UF, LF, HF, TOT ratings), they were to pick one of the letters that they believed was the first letter of the name of the pictured object. Even if they had no idea, they were asked to guess. After the first letter was selected, participants were then asked to guess how many syllables were in the target word, using the numbers one 
through five. Participants were allowed as much time as needed to make both the letter and syllable selection.

A recognition test was included on all trials where the target word was not retrieved. The experimenter read the correct target aloud, and participants orally indicated whether or not they recognized the object name ("yes" or "no"). This simple identification test is standard in TOT research (Brown \& Nix, 1996; Gollan \& Acenas, 2004; Gollan \& Brown, 2006) primarily to exclude those trials where the participant's intended target does not match the one provided by the experimenter (Schwartz, 2002; Schwartz \& Smith, 1997; Schwartz et al., 2000; Smith et al., 1994). Our purpose in using this test was not for exclusionary purposes, but as a simple verification that participants were using the response scale in an appropriate fashion.

To summarize the procedure, the target word object cue was first presented. If the participant provided the correct word aloud, the experimenter then proceeded to the next word cue. If the participant failed to retrieve the target word, they then went through the following steps (in order): (1) indicate how confident they were that they knew the object name, (2) select its first letter from among a set of three, (3) provide a syllable number guess (from one to five), and (4) respond "yes" or "no" to whether the target word spoken by the experimenter was familiar (UF, LF, HF) or whether the word was the one sought after during their TOT. The experimenter recorded all responses and controlled the trial-by-trial presentation procedure. Two practice trials were given, to ensure that participants understood the procedure. It should be noted that participants occasionally volunteered the object name after selecting the correct letter cue. They were not formally instructed to do so, and the experimenter recorded all such responses.

\section{Results}

For all analyses, an alpha level of .05 was used, unless otherwise stated. Participants correctly retrieved a mean of 75.0 target words $(59 \%)(S D=22.0)$, with $8.0 \mathrm{UF}(S D=5.5)$, 14.5 LF $(S D=9.0), 16.2 \mathrm{HF}(S D=11.8)$, and 13.5 TOT $(S D=$ 9.1) trials. As was expected, target-word recognition probability increased significantly across confidence levels: UF $=$ $.50(S D=.24), \mathrm{LF}=.80(S D=.13), \mathrm{HF}=.88(S D=.13)$ and $\mathrm{TOT}=.96(S D=.06), F(3,90)=38.72, M S E=.02 . \eta^{2}=.70$. The linear trend was significant, $F(1,17)=52.55, M S E=.04$, $\eta^{2}=.76$, confirming that participants were using recognition evaluations appropriately. More specifically, they admitted knowing only half of those words rated UF, even though they knew that all object labels provided were correct.

\section{First-letter identification}

Mean correct first-letter selection (Fig. 1) differed significantly across confidence levels, $F(3,90)=9.27, M S E=.04$ $\eta^{2}=.23$, and the increase across confidence level was confirmed by a significant linear trend, $F(1,30)=15.09$, $M S E=.06, \eta^{2}=.34$ (6 participants were excluded due to missing data in one or more confidence rating categories). More important, correct letter selection was significantly above chance (.33) at all confidence levels ${ }^{1}$ : UF, $t(19)=$ 2.63, $S E=.05$; LF, $t(22)=4.00, S E=.04$; HF, $t(21)=6.04$, $S E=.04$; TOT, $t(21)=7.06, S E=.05$.

Interestingly, letter selection accuracy appears to bias subsequent recognition evaluation, with target word recognition significantly higher following correct versus incorrect (respectively) letter guesses at each confidence level: UF, .61 versus $.44, t(30)=3.09, S E=.06 ; L F, .82$ versus .65 , $t(35)=3.77, S E=.04 ; \mathrm{HF}, .90$ versus $.81, t(36)=2.06, S E=$ .04 ; TOT, .96 versus $.85, t(28)=2.23, S E=.05$. Our interpretation is that if a participant selects " $\mathrm{S}$ " and then finds out that the target word is "stingray," they may assume

\footnotetext{
${ }^{1}$ A reviewer noted the possibility that participants could employ a letter choice strategy to boost their chance level of correct letter selection above $33 \%$, in the absence of any implicit knowledge of the word features. Given that the correct letter always occupies the middle frequency position in the set of five used for the triad generation, there is only one of the six possible triad combinations in which the target word's first letter has the lowest word generation frequency. If a participant were sensitive to first-letter word frequency and eliminated the one letter in the triad to which they had most difficulty generating words before choosing between the remaining two letters, such a strategy would increase their probability of guessing the correct letter from $33 \%$ to $50 \%$. They could also do the reverse, and eliminate the one letter in the triad that they had the least difficult generating words to and then select a letter from the remaining two. This would again boost their chance performance to $50 \%$.

Whereas such a strategy is possible, it is predicated on several assumptions: (1) many/all participants developed a strategic approach to letter selection, and (2) participants can successfully discriminate which letter is more/less frequently occurring at the beginning of words. These assumptions seem doubtful, for several reasons. First, letter selection was not the primary focus of the participants' performance (which was word retrieval) but, rather, a secondary response after the initial "failure" to retrieve. Second, according to the experimenter (who sat with each participant throughout the procedure), these selections were made too rapidly to support speculation that participants engaged in complex problem-solving strategy: attempt to generate words beginning with each of the three letters, eliminate the one letter that was easiest/hardest to generate candidate words from, and then select from the remaining two letters.

Finally, and perhaps most importantly, if participants were to use any strategy, it would make more sense to use a letter selection rather than a letter elimination approach. More specifically, identify the letter with the highest (or lowest) frequency of occurrence at the beginning of words as the response selection, rather than eliminate highest/lowest letters prior to making the choice. The letter selection strategy is more parsimonious, since it involves only one step: identify the highest probability letter. In contrast, the letter elimination strategy involves two steps: identify the highest/lowest probability first letter, then choose one of the remaining letters. If this selection (rather than elimination) strategy were followed, then the chance probability of being correct would actually decrease from $33 \%$ to $18 \%$, because the target letter would have the highest (or lowest) frequency in only one of the six possible letter triad combinations.
} 


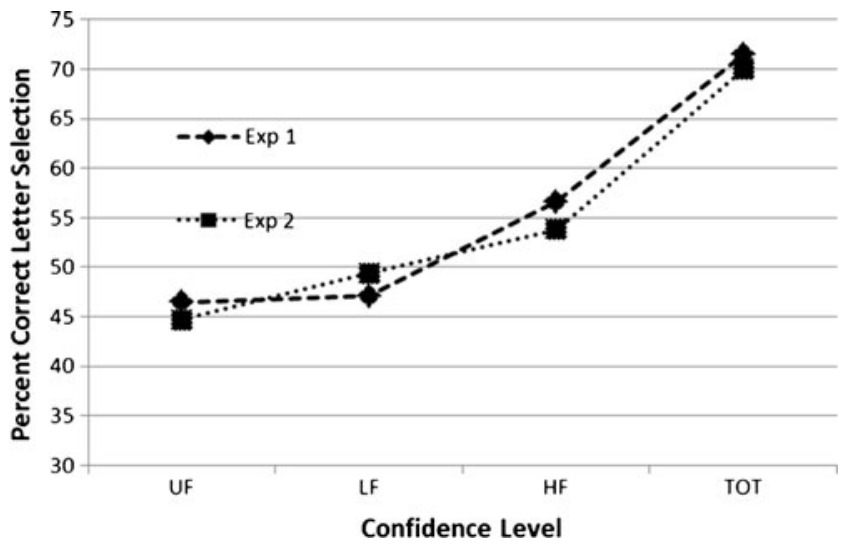

Fig. 1 Mean percent correct letter selection as a function of confidence level, for Experiments 1 and 2

that they had possessed implicit knowledge of the target word. This outcome implies that in studies where word attribute identification is encouraged (Brown \& Nix, 1996; Caramazza \& Miozzo 1997; Gollan \& Acenas, 2004), subsequent recognition evaluations may be compromised by the participants' guessing accuracy.

\section{Letter-cued retrieval}

Although participants were not given specific instructions regarding this, the act of identifying the correct first letter triggered successful retrieval in most of the participants (32 of 37). The probability of such "spontaneous retrievals" following correct first-letter guesses is presented in Fig. 2. Performance differed significantly across confidence level, $F(3,90)=10.27, M S E=.03 \eta^{2}=.28$, and the linear increase was significant, $F(1,30)=22.13, M S E=.03$, $\eta^{2}=.42$.

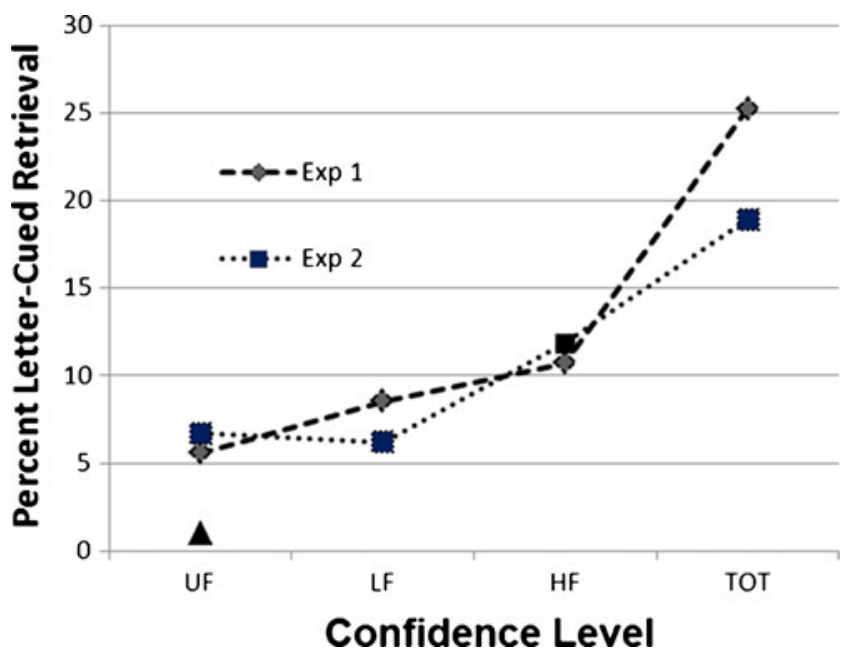

Fig. 2 Mean percent target word retrieval following correct letter selection as a function of confidence level, for Experiments 1 and 2

\section{Syllable number}

Excluding trials where the first-letter selection triggered target retrieval ( $15 \%$; see above), there was essentially no difference in syllable accuracy across confidence levels, $F<$ 1 (see Fig. 3). Chance levels cannot be accurately determined for our syllable guesses (Brown \& McNeill, 1966; Koriat \& Lieblich, 1974), but given that most target words $(77 \%)$ and guesses $(88 \%)$ were either two or three syllables, it would be difficult to argue that our $41 \%$ average could be considered above chance.

This syllable outcome is surprising, given the number of prior studies where some syllable knowledge appears to be available during TOTs (Brown, 2012). To explore this matter further, syllable guess error data were evaluated more closely. If participants have some sense of syllabic length, the deviation between actual and guessed syllable number should be lower at higher confidence levels. Defining small errors as 1 syllable off (either direction) and large as $2+$ syllables off, there was no significant difference in syllable guess accuracy across confidence level for either small errors $(\mathrm{UF}=.47, \mathrm{LF}=.45, \mathrm{HF}=.48, \mathrm{TOT}=.40)$ or large errors $(\mathrm{UF}=.13, \mathrm{LF}=.14, \mathrm{HF}=.12$, $\mathrm{TOT}=.18), F \mathrm{~s}<1$. Incidentally, target word retrieval following a correct syllable guess occurred on only one occasion.

\section{Discussion}

The central finding of Experiment 1 is above-chance firstletter accuracy across all confidence levels. In addition, there is a linear increase in accuracy from the lowest (UF) to the highest (TOT) category. A surprising finding is that selecting the correct letter triggers target word retrieval among most participants and at all confidence levels, despite

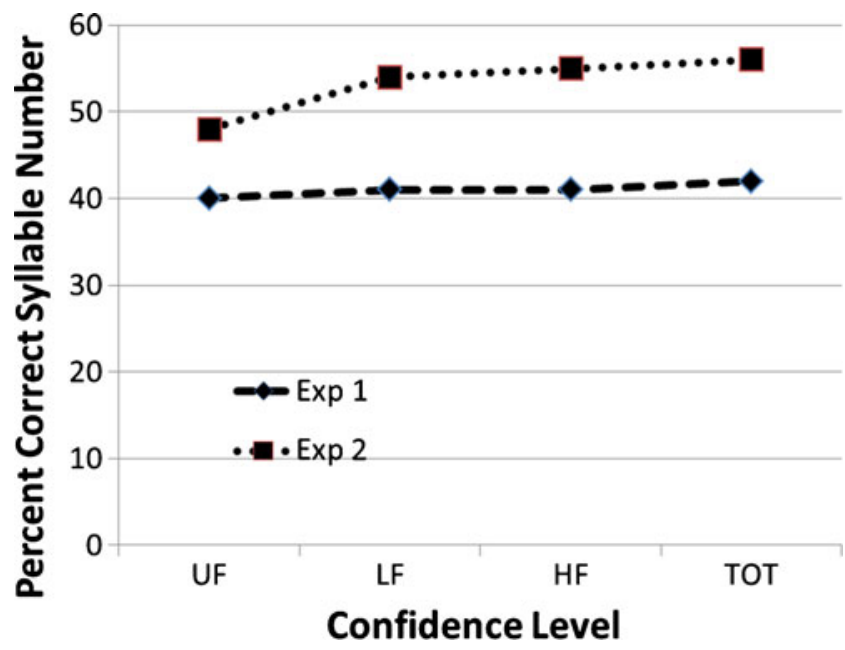

Fig. 3 Mean percent correct syllable number identification as a function of confidence level, for Experiments 1 and 2 
no instructions to do so. Furthermore, this rate of spontaneous retrievals progressively increases across confidence categories.

There is scant evidence of syllable number awareness at any confidence level. In fact, it is no higher during TOTs (.42) than across the DK categories (UF, LF, HF) (.41). Although Brown and McNeill's (1966) participants were impressively adept at guessing syllabic length for TOTs ( $60 \%$ ), the bulk of findings since then do not provide strong evidence for syllable accessibility (cf. Brown, 2012). Before drawing conclusions from these data about syllable number accessibility, a possible item selection bias needs to be addressed. Target word recall probability following correct letter selection increased systematically from .06 for UF to .15 for TOT (see Fig. 2), and these words were not available for the subsequent syllable guesses. Thus, the pool of items remaining to be evaluated for syllable knowledge may be progressively less familiar across increasing confidence categories. To address this, Experiment 2 reversed the procedure, requiring participants to guess the syllable first and the first letter second. The syllable selection task was also simplified by using only two- or three-syllable target words so that chance levels could be established.

\section{Experiment 2}

\section{Method}

\section{Participants}

A new sample of 72 students from sections of Introductory Psychology at Southern Methodist University volunteered to participate in the study and received extra course credit as incentive.

\section{Materials}

From the 127 target words used in Experiment 1, 43 twosyllable and 43 three-syllable target words were selected for Experiment 2 (see Table 1). The presentation order of object cues was block randomized, such that three target names of each syllabic length were represented within each successive six object cues.

\section{Procedure}

The procedure was changed in two ways from Experiment 1. First, when participants could not recall the target word, their familiarity rating was followed first by a syllable number guess ("two" or "three") and then by a first letter selection. Second, participants were further informed that half of the target words were of each syllabic length.

\section{Results}

Participants correctly retrieved a mean of 51.8 target words $(52 \%)(S D=13.4)$ and classified 5.2 as UF $(S D=4.8), 8.5$ as $\operatorname{LF}(S D=5.4), 11.3$ as HF $(S D=5.1)$, and 9.3 as TOT $(S D=6.1)$. As in Experiment 1 , recognition probability increased significantly across confidence level: UF $=.44$ $(S D=.29), \mathrm{LF}=.65(S D=.24), \mathrm{HF}=.87(S D=.12)$, and TOT $=.95(S D=.14)$. The overall difference was significant, $F(3,126)=49.59, M S E=.04, \eta^{2}=.54$, as well as the linear increase, $F(1,42)=122.25, M S E=.05, \eta^{2}=.74$.

\section{Syllable number}

The percent correct syllable guess accuracy (see Fig. 3) was above chance (.50) for TOT (.56), $t(70)=2.06, S E=.03$, and HF (.55), $t(71)=2.93, S E=.02$, but not for UF (.48), $t(70)=1.47, S E=.02$, and LF $(.54), t(43)=0.59, S E=.04$. However, an ANOVA yielded no significant overall difference across the four confidence categories, $F<1$, and no significant linear trend, $F<1$. Syllable guessing accuracy was unimpressive and, at best, only $6 \%$ above chance. As in Experiment 1, target retrieval following a correct syllable guess was rare (seven occasions).

\section{Letter identification}

First-letter accuracy (see Fig. 1) was again significantly above chance $(.33)$ at each confidence level: UF, $t(43)=$ $2.54, S E=.04 ; \mathrm{LF}, t(70)=5.50, S E=.03 ; \mathrm{HF}, t(71)=10.91$, $S E=.02$; TOT, $t(70)=14.52, S E=.03$. The overall difference across confidence level was significant, $F(3,126)=$ 9.57, $M S E=.05, \eta^{2}=.19$, as well as the linear increase across confidence conditions, $F(1,42)=20.07, M S E=.06$, $\eta^{2}=.32$.

As in Experiment 1, recognition probability was higher following correct versus incorrect letter selection, and significantly so for all levels except UF: UF (.50 vs. .41), $t(32)=$ $1.00, S E=.05$; LF (.71 vs. .62), $t(59)=2.01, S E=.05$; HF (.92 vs. .81$), t(69)=3.96, S E=.05$; TOT (.98 vs. .90$), t$ $(56)=3.44, S E=.04$. In contrast, recognition accuracy did not differ between correct and incorrect syllable selection trials at any confidence level: UF (.49 vs. .42), $t(33)=0.94, S E=.07$; LF (.69 vs. .61), $t(65)=1.51, S E=.05$; HF (.87 vs. .86$)$, $t(69)=0.46, S E=.22$; TOT (.96 vs. .96), $t(58)=0.02, S E=.01$.

\section{Letter-cued retrieval}

As was observed in Experiment 1, the majority of participants (67 of 72) had the target word come to mind following correct letter selection, even though they were not given instructions concerning this. Such spontaneous target word retrieval following correct letter selection (see Fig. 2) 
differed significantly across confidence level, $F(3,96)=$ 3.52, $M S E=.02, \eta^{2}=.10$, and the linear increase was significant, $F(1,32)=7.29, M S E=.03, \eta^{2}=.19$.

\section{Discussion}

The outcome of Experiment 2 extends the reliability of the outcome from Experiment 1. More specifically, the ability of participants to detect the first letter of the unavailable target word is significantly above chance across all confidence levels and increases in a linear fashion. Furthermore, correct first-letter selection triggers correct word retrieval on some occasions at each of the confidence levels and at a progressively increasing rate. Unlike letter detection, the ability to guess syllable number above chance levels is limited to only the higher confidence levels (HF and TOT), and even these levels are not impressive.

\section{General discussion}

The primary outcome of the present research is that abovechance identification of the initial letter of unavailable target words is not reserved to TOTs but exists at all confidence levels. Summed across experiments, first-letter detection ability exceeds chance ( $33 \%$ ) by $12 \%$ for UFs, $16 \%$ for LFs, $21 \%$ for HFs, and $37 \%$ for TOTs. This confirms and extends Koriat and Lieblich's (1974) finding of first-letter access during DK trials but refines this by showing that this sensitivity exists at both high and low levels of DK, in an appropriately graded fashion. Clearly, Koriat and Lieblich's above-chance performance during DKs is not driven by a subset of weak TOTs that happen to be demoted by the participant to a lesser confidence level (DK). Rather, participants consistently have some success at identifying the first letter, even when they claim to be unfamiliar with the target word.

In stark contrast to the strong and consistent evidence for first-letter access, there is only weak evidence for syllable access. In both experiments, syllable information did not differ across confidence levels and was above chance only at higher confidence levels (HF, TOT) in Experiment 2. Even then, it was unimpressive at $6 \%$ (or less) above chance. Our modest evidence indicates that perhaps a general (long/short), rather than specific (syllable number), sense of word length may be accessible for some higher-confidence unrecalled words. Such modest evidence is comparable to Hanley and Chapman's (2008) finding that accuracy in identifying word number in unretrieved celebrity names (two words, Gwyneth Paltrow; three words, Billy Bob Thornton) was only $7 \%$ higher during TOTs $(56 \%)$ than during DKs (49\%). Interestingly, their DK performance was clearly at chance, similar to our UF and LF items in Experiment 2.
A common TOT resolution strategy in anecdotal reports involves alphabet search (Burke et al., 1991; Cohen \& Faulkner, 1986; Finley \& Sharp, 1989; Gruneberg \& Monks, 1974; Reason \& Lucas, 1984), and prior research confirms that providing the actual first letter can help participants resolve a TOT (Brennan, Baugley, Bright, \& Bruce, 1990; Finley \& Sharp, 1989; Freedman \& Landauer, 1966; Gruneberg \& Monks, 1974; Heine, Ober, \& Shenaut, 1999). The present procedure better models the real-life alphabet search process, in that the participants must select the first letter rather than having it provided. Target word retrieval cued by successful first-letter selection occurred for nearly one out of every four TOTs ( $23 \%$ ), as compared with 1 in 12 DKs ( $8 \%$ ), suggesting that excitation level of the initial phonological node is much closer to threshold during a TOT. Furthermore, it was a nearly universal experience, with $91 \%$ of participants across experiments experiencing such spontaneous retrievals.

As was suggested earlier, although Cleary's RWI research was limited to episodic memory tasks, the same mechanisms may apply to assessing the availability of structural word information from semantic memory. Furthermore, such implicit availability may influence familiarity assessments manifest in confidence ratings and TOT evaluations. Cleary has drawn such a comparison between RWI and TOT experiences (Cleary, 2006; Cleary \& Reyes, 2009; Cleary \& Specker, 2007; Cleary et al., 2010), in that awareness of a word's attributes stored in semantic memory may be similar to feeling that a word appeared in the prior list. If a rough parallel may be drawn to the present outcome, we similarly discovered that access to lexical information regarding unretrieved words was available in both TOT and DK states.

The two main theoretical positions regarding TOTs can easily predict above-chance first-letter access during TOT states. However, neither makes a straightforward prediction about first-letter access during DK states. The most widely supported view of TOT etiology is the transmission deficit hypothesis, or TDH (Burke et al., 1991). During routine word retrieval, the level of priming passed from the semantic to the phonological nodes is variable, such that weaker connections transmit less excitation. Above-threshold phonological activation results in word production, but subthreshold activation leads to a TOT because the availability of phonological features (e.g., first letter) leads to a high-confidence prediction of imminent word accessibility. Although TDH does not speculate about what happens during DK states, a reasonable extension of TDH is that some potential first-letter access may exist across all levels of retrieval confidence. If excitability spreads from the semantic to phonological nodes in a graded fashion, evidence of target word first-letter awareness may be found even at low confidence levels. In fact, variation in the degree of phonological activation may be what drives the confidence ratings. Additional support for TDH 
comes from the unanticipated finding that retrieval is occasionally triggered by correct letter selection. That this occurred more often during TOT than during DK (UF, LF, HF) suggests that the phonological node is in an especially energized state during TOTs and that vocalizing the first letter may supply an additional boost needed to exceed threshold.

The metacognitive interpretation of TOTs (Schwartz, 2002) cannot easily accommodate the above-chance firstletter identification across all confidence levels found in the present study. Under this theoretical position, it is conceivable that information about the target word comes to mind during HF and TOT states, which both drives these ratings and accounts for above-chance letter accuracy. However, the accessibility interpretation would not imply that target word features are activated during LF or UF ratings (Koriat, 1994). Otherwise, these ratings logically would be boosted to HF or TOT level. Yet participants were consistently above chance for letter identification in both UF and LF states.

While we propose that some degree of automatic activation of the nonretrieved word node underlies our findings of first-letter identification success, Koriat and Lieblich (1974) have suggested an alternative mechanism involving class detection, where one can guess features of a word on the basis of general knowledge about common structural properties of words in a specific semantic category. For example, the distribution of first-letter probabilities for medical terms differs from that for words describing geological features. Koriat and Lieblich speculated that class detection alone could account for first-letter guessing accuracy in both DK and TOT states. In the present outcome, class differentiation should be constant across UF, LF, and HF states, given that the semantic category of the pictured object can be ascertained equally well in each condition. However, the reliably increasing trend in letter selection accuracy across confidence levels (see Fig. 1) does not support the class detection interpretation.

The studies in this article have broader implications for the availability of word knowledge in the absence of word access. We demonstrated that individuals can access a central orthographic/phonological feature (first letter) reliably above chance, and this dovetails with previous research on successful access to semantic and contextual word attributes in the absence of identification. With respect to semantic aspects of inaccessible words, research has confirmed that the semantic differential dimensions of evaluation (Eysenck, 1979; Koriat et al., 2003; Yavuz \& Bousfield, 1959), activity (Koriat et al., 2003), and potency (Koriat et al., 2003) can be identified at above-chance levels for inaccessible words. Furthermore, contextual dimensions associated with the word's prior presentation are also available above chance for unrecalled words, and these include speaker's voice (Kurilla \& Westerman, 2010), processing during input (imagery; pleasantness) (Starns, Hicks, Brown, \& Martin, 2008), and input list position (Kurilla \& Westerman, 2010) (but see Schacter \& Worling, 1985, and Cook, Marsh, and Hicks, 2006, for exceptions).

Perhaps the most remarkable finding in the present study is that implicit knowledge was consistently evident even when the target word was declared to be unknown. Related to this are findings that semantic features can be identified for words that participants cannot define, or assess to be unknown (Durso \& Shore, 1991; Eysenck, 1979; Koriat et al., 2003; Nelson, Fehling, \& Moore-Glascock, 1979; Shore \& Durso, 1990; Shore \& Kempe, 1999; Whitmore, Shore, \& Smith, 2004; Yavuz \& Bousfield, 1959). Shore and Durso had participants separate words into three groups: (1) known (could provide a definition), (2) frontier (had encountered before but did not know meaning), and (3) unknown (had no idea whether it was a word). Participants showed above-chance awareness of semantic features for both frontier ( $26 \%$ above chance) and unknown (20\% above chance) words, as reflected in the identification of the appropriate sentence context (Durso \& Shore, 1991; Shore \& Kempe, 1999). Our present finding extends this to structural information, showing that the first letter of a word is also available regardless of whether the word is assessed as frontier (our LF and HF) or unknown (our UF).

Wagovich and Newhoff (2004) suggested that such implicit semantic word knowledge provides a lexical platform upon which complete word knowledge is incrementally constructed. This happens outside of our direct awareness, accrues unintentionally through repeated experiences, and can be found even after one prior exposure. It seems a reasonable implication of the present research that phonological and orthographic aspects of words may similarly be constructed in a slow, accretive manner. First-letter recognition for words assessed as unfamiliar (UF) or low familiar (LF) appears to provide evidence that implicit knowledge of words may be built from minimal prior exposure. In fact, the present outcome supports speculation that the line between implicit and explicit memory may not be all that distinct (Dew \& Cabeza, 2011) and may be graded in a manner suggested by our finding of a systematic increase in firstletter knowledge from no knowledge (UF) to imminent access (TOT).

In summary, we found that first-letter information is available above chance at all confidence levels for unretrieved targets, from TOT down to unfamiliar (UF). Evidence for syllable access is weak and only above chance with high DKs (HF) or TOTs (Experiment 2). Our outcome also invites an extension of RWI as a mechanism possibly involved during routine word retrieval (Cleary, 2002). Some component(s) of a word's representation may be activated and detectable, even in the absence of identification or retrieval. This outcome complements prior research showing access to semantic 
dimensions of unfamiliar words and suggests that implicit access to structural features can occur as well.

Author Notes We greatly appreciate the help of Jill Gregory and Alexandra Lippas in testing the participants for these studies and Lindy Loneman for comments on an earlier draft of the manuscript. Portions of this study were presented at the 2009 Psychonomic Society Convention in Boston, MA.

\section{References}

Bahrick, H. P. (2008). Thomas O. Nelson: His life and comments on implications of his functional view of metacognitive memory monitoring. In J. Dunlosky \& R. A. Bjork (Eds.), Handbook of metamemory and memory (pp. 1-7). New York: Psychology Press.

Biedermann, B., Ruh, N., Nickels, L., \& Coltheart, M. (2008). Information retrieval in tip of the tongue states: New data and methodological advances. Journal of Psycholinguistic Research, 37, 171-198.

Brennen, T., Baguley, T., Bright, J., \& Bruce, V. (1990). Resolving semantically induced tip-of-the-tongue states for proper nouns. Memory and Cognition, 18, 339-347.

Brown, A. S. (1991). A review of the tip of the tongue phenomenon. Psychological Bulletin, 109, 204-223.

Brown, A. S. (2012). Tip of the tongue states. New York: Psychology Press.

Brown, A. S., \& Nix, L. A. (1996). Age-related changes in the tip-ofthe-tongue experience. The American Journal of Psychology, 109, 79-91.

Brown, R., \& McNeill, D. (1966). The "tip of the tongue" phenomenon. Journal of Verbal Learning and Verbal Behavior, 5, 325-337.

Burke, D. M., MacKay, D. G., Worthley, J. S., \& Wade, E. (1991). On the tip of the tongue: What causes word finding failures in young and older adults? Journal of Memory and Language, 30, 542579 .

Caramazza, A., \& Miozzo, M. (1997). The relation between syntactic and phonological knowledge in lexical access: Evidence from the "tip-of-the-tongue" phenomenon. Cognition, 64, 309-343.

Cleary, A. M. (2002). Recognition with and without identification: Dissociative effects of meaningful encoding. Memory \& Cognition, 30, 758-767.

Cleary, A. M. (2006). Relating familiarity-based recognition and the tip-of-the tongue phenomenon: Detecting a word's recency in the absence of access to the word. Memory \& Cognition, 34, 804 816

Cleary, A. M. (2008). Recognition memory, familiarity, and déjà vu experiences. Current Directions in Psychological Science, 17, 353-357.

Cleary, A. M., Konkel, K. E., Nomi, J. S., \& McCabe, D. P. (2010). Odor recognition without identification. Memory \& Cognition, $38,452-460$.

Cleary, A. M., Langley, M. M., \& Seiler, K. R. (2004). Recognition without picture identification: Geons as components of the pictorial memory trace. Psychonomic Bulletin \& Review, 11, 903-908.

Cleary, A. M., \& Reyes, N. L. (2009). Scene recognition without identification. Acta Psychologica, 131, 53-62.

Cleary, A. M., \& Specker, L. E. (2007). Recognition without face identification. Memory \& Cognition, 35, 1610-1619.

Cohen, G., \& Faulkner, D. (1986). Memory for proper names: Age differences in retrieval. British Journal of Developmental Psychology, 4, 187-197.
Cook, G. I., Marsh, R. L., \& Hicks, J. L. (2006). Source memory in the absence of successful cued recall. Journal of Experimental Psychology: Learning, Memory, and Cognition, 32, 828-835.

Dew, I. T. Z., \& Cabeza, R. (2011). The porous boundaries between explicit and implicit memory: Behavioral and neural evidence. Annals of the New York Academy of Sciences, 1224, 174-190.

Durso, F. T., \& Shore, W. J. (1991). Partial knowledge of word meanings. Journal of Experimental Psychology. General, 120, 190-202.

Eysenck, M. W. (1979). The feeling of knowing a word's meaning. British Journal of Psychology, 70, 243-251.

Finley, G. E., \& Sharp, T. (1989). Name retrieval by the elderly in the tip-of-the- tongue paradigm: Demonstrable success in overcoming initial failure. Educational Gerontology, 15, 259-265.

Freedman, J. L., \& Landauer, T. K. (1966). Retrieval of long-term memory: "Tip-of-the-tongue" phenomenon. Psychonomic Science, 4, 309-310.

Gollan, T. H., \& Acenas, L. R. (2004). What is a TOT? Cognate and translation effects on tip of-the-tongue states in Spanish-English and Tagalog-English bilinguals. Journal of Experimental Psychology: Learning, Memory, and Cognition, 30, 246-269.

Gollan, T. H., \& Brown, A. S. (2006). From tip-of-the-tongue (TOT) data to theoretical implications: When more TOTs means better retrieval. Journal of Experimental Psychology. General, 135, 462-483.

Gruneberg, M. M., \& Monks, J. (1974). 'Feeling of knowing' and cued recall. Acta Psychologica, 38, 257-265.

Hanley, J. R., \& Chapman, E. (2008). Partial knowledge in a tip-of-thetongue state about two- and three-word proper names. Psychonomic Bulletin \& Review, 15, 156-160.

Heine, M. K., Ober, B. A., \& Shenaut, G. K. (1999). Naturally occurring and experimentally induced tip-of-the-tongue experiences in three adult age groups. Psychology and Aging, 14, $445-457$.

Jönsson, F. U., \& Olsson, M. J. (2003). Olfactory metacognition. Chemical Senses, 28, 651-658.

Jönsson, F. U., Tchekhova, A., Lonner, P., \& Olsson, M. J. (2005). A metamemory perspective on odor naming and identification. Chemical Senses, 30, 353-365.

Koriat, A. (1993). How do we know that we know? The accessibility model of the feeling of knowing. Psychological Review, 100, 609-639.

Koriat, A. (1994). Memory's knowledge of its own knowledge: The accessibility account of the feeling of knowing. In J. Metcalfe \& A. P. Shimamura (Eds.), Metacognition: Knowing about knowing (pp. 115-136). Cambridge: The MIT Press.

Koriat, A., Levy-Sadot, R., Edry, E., \& de Marcas, S. (2003). What do we know about what we cannot remember? Accessing the semantic attributes of words that cannot be recalled. Journal of Experimental Psychology: Learning, Memory, and Cognition, 29, 1095-1105.

Koriat, A., \& Lieblich, I. (1974). What does a person in a "TOT" state know that a person in a "don't know" state doesn't know. Memory \& Cognition, 2, 647-655.

Kostic, B., \& Cleary, A. M. (2009). Song recognition without identification: When people cannot "name that tune" but can recognize it as familiar. Journal of Experimental Psychology: General, 138, $146-159$

Kurilla, B. P., \& Westerman, D. L. (2010). Source memory for unidentified stimuli. Journal of Experimental Psychology: Learning, Memory, and Cognitiion, 36, 398-410.

Mayzner, M. S., \& Tresselt, M. E. (1965). Tables of single-letter and digram frequency counts for various word-length and letterposition combinations. Psychonomic Monograph Supplements, 1 (Whole No. 2), 13-32.

Metcalfe, J., Schwartz, B. L., \& Joaquim, S. G. (1993). The cuefamiliarity heuristic in metacognition. Journal of Experimental Psychology: Learning, Memory, and Cognition, 19, 851-864. 
Miozzo, M., \& Caramazza, A. (1997). Retrieval of lexical-syntactic features in tip- of-the-tongue states. Journal of Experimental Psychology: Learning, Memory, and Cognition, 23, 1410-1423.

Naito, M., \& Komatsu, S. (1989). Effects of conceptually driven processing on perceptual identification. Japanese Psychological Research, 31, 45-56.

Nelson, T. O., Fehling, M. R., \& Moore-Glascock, J. (1979). The nature of semantic savings for items forgotten from long-term memory. Journal of Experimental Psychology. General, 108, $225-250$.

Peynircioğlu, Z. F. (1990). A feeling-of-recognition without identification. Journal of Memory and Language, 29, 493-500.

Reason, J. T., \& Lucas, D. (1984). Using cognitive diaries to investigate naturally occurring memory blocks. In J. Harris \& P. E. Morris (Eds.), Everyday memory, actions, and absent mindedness (pp. 53-70). London: Academic.

Schacter, D. L., \& Worling, J. R. (1985). Attribute information and the feeling-of knowing. Canadian Journal of Psychology, 39, 467-475.

Schwartz, B. L. (2002). Tip-of-the-tongue states: Phenomenology, mechanism, and lexical retrieval. Mahwah: Erlbaum Associates.

Schwartz, B. L. (2008). Working memory load differentially affects tip-of-the-tongue states and feeling-of-knowing judgments. Memory \& Cognition, 36, 9-19.

Schwartz, B. L., \& Smith, S. M. (1997). The retrieval of related information influences tip-of-the-tongue states. Journal of Memory and Language, 36, 68-86.
Schwartz, B. L., Travis, D. M., Castro, A. M., \& Smith, S. M. (2000). The phenomenology of real and illusory tip-of-the-tongue states. Memory and Cognition, 28, 18-27.

Shore, W. J., \& Durso, F. T. (1990). Partial knowledge in vocabulary acquisitions: General constraints and specific detail. Journal of Educational Psychology, 82, 315-318.

Shore, W. J., \& Kempe, V. (1999). The role of sentence context in accessing partial knowledge of word meaning. Journal of Psycholinguistic Research , 28, 145-163.

Smith, S. M., Balfour, S. P., \& Brown, J. M. (1994). Effects of practice on tip-of-the-tongue states. Memory, 2, 31-49.

Snodgrass, J. G., \& Vanderwart, M. (1980). A standardized set of 260 pictures: Norms for name agreement, image agreement, familiarity, and visual complexity. Journal of Experimental Psychology: Human Learning and Memory, 6, 174-215.

Starns, J. J., Hicks, J. L., Brown, N. L., \& Martin, B. A. (2008). Source memory for unrecognized items: Predictions from multivariate signal detection theory. Memory \& Cognition, 36, 1-8.

Wagovich, S. A., \& Newhoff, M. (2004). The single exposure: Partial word knowledge growth through reading. American Journal of Speech-Language Pathology, 13, 316-328.

Whitmore, J. M., Shore, W. J., \& Smith, P. H. (2004). Partial knowledge of word meanings: Thematic and taxonomic representations. Journal of Psycholinguistic Research , 33, 137-164.

Yavuz, H. S., \& Bousfield, W. A. (1959). Recall of connotative meaning. Psychological Reports, 5, 319-320. 